Influence of annealing conditions on the formation of regular lattices of voids and Ge quantum dots in an amorphous alumina matrix

This article has been downloaded from IOPscience. Please scroll down to see the full text article.

2012 Nanotechnology 23405605

(http://iopscience.iop.org/0957-4484/23/40/405605)

View the table of contents for this issue, or go to the journal homepage for more

Download details:

IP Address: 193.137.16.117

The article was downloaded on 21/09/2012 at 18:12

Please note that terms and conditions apply. 


\title{
Influence of annealing conditions on the formation of regular lattices of voids and Ge quantum dots in an amorphous alumina matrix
}

\author{
S R C Pinto ${ }^{1}$, M Buljan ${ }^{2}$, L Marques ${ }^{1}$, J Martín-Sánchez ${ }^{3}$, O Conde ${ }^{4}$,

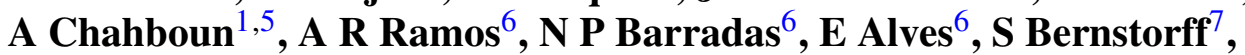 \\ J Grenzer ${ }^{8}$, A Mücklich ${ }^{8}$, M M D Ramos ${ }^{1}$ and M J M Gomes ${ }^{1}$ \\ ${ }^{1}$ Centre of Physics and Physics Department, University of Minho, Braga, 4710-057, Portugal \\ ${ }^{2}$ Ruđer Bošković Institute, Bijenička cesta 54, 10000 Zagreb, Croatia \\ ${ }^{3}$ Laser Processing Group, Instituto de Óptica, CSIC, C/Serrano 121, E-28006 Madrid, Spain \\ ${ }^{4}$ Department of Physics, University of Lisbon, P-1749016 Lisbon, Portugal \\ ${ }^{5}$ Physics Department, FST Tanger, BP 416 Tangier, Morocco \\ ${ }^{6}$ Nuclear and Technological Institute (ITN), EN10, 2686-953 Sacavém, Portugal \\ ${ }^{7}$ Sincrotrone Trieste, SS 14 km163, 5, I-34012 Basovizza, Italy \\ ${ }^{8}$ Helmhotz-Zentrum Dresden-Rossendorf, Dresden, Germany \\ E-mail: sarapinto@ fisica.uminho.pt
}

Received 31 May 2012, in final form 8 August 2012

Published 20 September 2012

Online at stacks.iop.org/Nano/23/405605

\begin{abstract}
In this work, the influence of air pressure during the annealing of Ge quantum dot (QD) lattices embedded in an amorphous $\mathrm{Al}_{2} \mathrm{O}_{3}$ matrix on the structural, morphological and compositional properties of the film is studied. The formation of a regularly ordered void lattice after performing a thermal annealing process is explored. Our results show that both the Ge desorption from the film and the regular ordering of the QDs are very sensitive to the annealing parameters. The conditions for the formation of a void lattice, a crystalline Ge QD lattice and a disordered QD lattice are presented. The observed effects are explained in terms of oxygen interaction with the Ge present in the film.
\end{abstract}

(Some figures may appear in colour only in the online journal)

\section{Introduction}

Semiconductor quantum dots (QDs) and nanocrystals have been intensively studied in recent years due to their unique and interesting physical properties for practical applications in optoelectronic, photovoltaic and photonic applications among others [1-6]. Si and Ge materials have received most of the attention due to their low cost, low toxicity, abundance in Earth resources and compatibility with state-of-the-art silicon technology [7]. Compared to $\mathrm{Si}, \mathrm{Ge}$ is gaining significance as a material for future nanoelectronic devices due to its higher carrier mobility, lower band-gap and higher quantum confinement [8]. On the other hand, high- $k$ dielectric materials such as $\mathrm{Al}_{2} \mathrm{O}_{3}$ are an excellent alternative to conventional $\mathrm{SiO}_{2}$ for the scaling of complementary metal oxide semiconductor (CMOS) technology [9]. In addition, $\mathrm{Al}_{2} \mathrm{O}_{3}$ is also an excellent construction material due to its high hardness, mechanical strength and stiffness. In particular, porous $\mathrm{Al}_{2} \mathrm{O}_{3}$ is of interest for various applications due to its excellent dielectric, mechanical and thermal properties, as well as its very low specific mass [10].

In order to make Ge CMOS devices feasible, it is necessary to understand and control the high- $k / \mathrm{Ge}$ interface properties $[11,12]$. The post-growth annealing process at high temperatures $\left(>800^{\circ} \mathrm{C}\right)$ is an important step to achieve Ge QDs embedded in dielectric host matrices [13-15]. However, 
Table 1. Average sizes of Ge QDs/voids and parameters of the QD/void lattice for the as-grown and annealed films calculated from GISAXS maps.

\begin{tabular}{lcccc}
\hline & & \multicolumn{3}{c}{ Annealed films } \\
\cline { 2 - 5 } Sample name & As-grown & $P_{1}=10^{-5} \mathrm{mbar}$ & $P_{2}=10^{-3} \mathrm{mbar}$ & $P_{3}=P_{\text {atm }}$ \\
\hline \multicolumn{5}{c}{ Average QD size $(\mathrm{nm})$} \\
\hline A & $4.6 \pm 0.4$ & $4.2 \pm 0.4$ & $4.2 \pm 0.4$ & $>5$ \\
B & $2.1 \pm 0.4$ & $2.0 \pm 0.4$ & $2.0 \pm 0.4$ & $>6$ \\
\hline \multicolumn{5}{c}{$a(\mathrm{~nm})$} \\
\hline A & $9.8 \pm 0.2$ & $9.8 \pm 0.2$ & $9.8 \pm 0.2$ & - \\
B & $6.2 \pm 0.2$ & $6.2 \pm 0.2$ & $6.2 \pm 0.2$ & - \\
\hline A & $11.4 \pm 0.2$ & $11.2 \pm 0.4$ & $10.8 \pm 0.2$ & - \\
B & $7.8 \pm 0.2$ & $7.8 \pm 0.4$ & $7.8 \pm 0.2$ & - \\
\hline
\end{tabular}

the thermodynamically unstable nature of Ge native oxides, which may be produced during the annealing treatments, can hamper the successful development of devices due to volatile germanium monoxide $(\mathrm{GeO})$ species desorption. In this regard, Ge redistribution and loss are strongly influenced by moisture contaminants $\left(\mathrm{H}_{2} \mathrm{O}, \mathrm{OH}, \mathrm{H}_{2}\right)$ that are originated from air humidity, wet cleaning chemicals or residual oxygen in the inert gases used during annealing treatments or deposition [16, 17]. In particular, $\mathrm{GeO}$ desorption from a high- $k / \mathrm{Ge}$ system has been demonstrated to deteriorate the surface and interface quality, which could eventually degrade the device performance [12]. However, the desorption of $\mathrm{Ge}$ QDs from Ge-rich oxide films after performing annealing processes has rarely been studied [18], especially when embedded in an $\mathrm{Al}_{2} \mathrm{O}_{3}$ matrix. Recently, we have reported the formation of regularly ordered Ge QD arrays (QD lattices) embedded in an amorphous $\mathrm{Al}_{2} \mathrm{O}_{3}$ matrix, which is very interesting for control of the properties of the material [19]. The regular ordering was achieved by co-deposition of a continuous $\left(\mathrm{Ge}+\mathrm{Al}_{2} \mathrm{O}_{3}\right)$ layer at a substrate temperature of $500{ }^{\circ} \mathrm{C}$. In another work, we have shown that annealing of the regularly ordered Ge QD lattices can result in the formation of regularly ordered void lattices in an $\mathrm{Al}_{2} \mathrm{O}_{3}$ matrix where the complete desorption of Ge from the film takes place [20]. The annealing conditions promote the diffusion of $\mathrm{Ge}$ atoms out of the matrix and voids remain at the places where Ge was initially present. Thus, the result of the annealing is the formation of a void lattice in the $\mathrm{Al}_{2} \mathrm{O}_{3}$ matrix with the same ordering as the initial Ge QD lattice.

In this work, we investigate in more detail the evolution of regular Ge QD lattices as a function of the annealing conditions leading to the formation of a void lattice or a crystalline Ge QD lattice. This study has been performed on films containing Ge QD lattices with different QD sizes and spatial QD distributions under different annealing pressures in air: from atmospheric pressure down to $10^{-5}$ mbar. We demonstrate a strong influence of the annealing pressure on the structural and compositional properties of the film. In particular, we study the evolution of the regular ordering of embedded nano-objects (Ge QDs and voids) and diffusion of Ge atoms out of the film. The investigated films show the same qualitative behavior for similar annealing conditions, independently of the initial QD lattice. Interestingly, a complete Ge desorption from the film leading to the formation of a void lattice is found for intermediate annealing pressures (about $10^{-3}$ mbar), whereas this Ge desorption from the film is avoided for other annealing pressures. A clear evolution of the QD lattice is observed with a complete destruction of the initially present regular ordering for an annealing performed at atmospheric pressure. The Ge out-diffusion from the film as a function of the annealing pressure is discussed.

\section{Experimental details}

\subsection{Sample preparation}

Thick films of Ge embedded in $\mathrm{Al}_{2} \mathrm{O}_{3}$ were grown using a commercial RF-magnetron Alcatel SCM 650 sputtering machine. $\mathrm{Al}_{2} \mathrm{O}_{3}(99.99 \%)$ and polycrystalline $\mathrm{Ge}(99.99 \%)$ were simultaneously co-sputtered to produce Ge doped films using an RF power of $50 \mathrm{~W}$. Low electrical resistivity (3-6 $\Omega \mathrm{cm}$ ) n-type $\operatorname{Si}(111) 2$ inch wafers were used as substrates. Prior to sputtering, a base pressure of $1 \times$ $10^{-6}$ mbar was reached inside the growth chamber and in situ argon plasma treatment was performed on the target and the substrates to clean their surfaces. Two kinds of sample were studied with different Ge QD sizes and lattice configurations (see table 1): large QD size ( $4.6 \pm 0.4 \mathrm{~nm}$, hereafter A) and small QDs size $(\sim 2.1 \pm 0.4 \mathrm{~nm}$, hereafter B). Film A was produced using a substrate temperature of $T_{\mathrm{S}}=500^{\circ} \mathrm{C}$ and Ar pressure of $P_{\mathrm{Ar}}=5 \times 10^{-3}$ mbar. Film B was produced using $T_{\mathrm{S}}=250^{\circ} \mathrm{C}$ and $P_{\mathrm{Ar}}=4 \times 10^{-3}$ mbar. Afterwards, the samples were subjected to an annealing treatment using a conventional furnace in air at $800^{\circ} \mathrm{C}$ for $1 \mathrm{~h}$ at different annealing pressures: $P_{1}=10^{-5} \mathrm{mbar}, P_{2}=10^{-3} \mathrm{mbar}, P_{3}=$ $P_{\mathrm{atm}}=1000$ mbar.

\subsection{Experimental characterizations}

The structural characterization of the films was performed by x-ray diffraction (XRD) in conventional $\theta-2 \theta$ geometry (Philips PW1710) using $\mathrm{Cu} \mathrm{K} \alpha$ radiation. The identification 

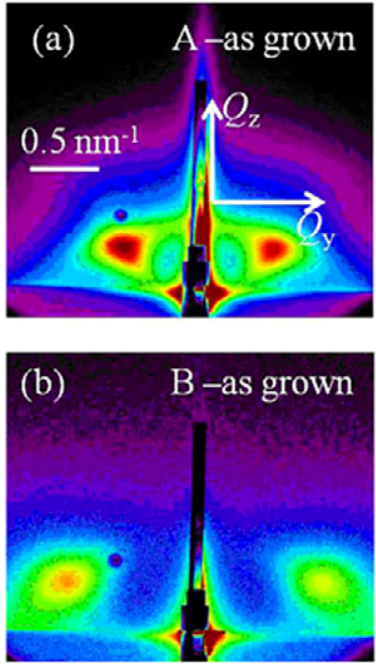
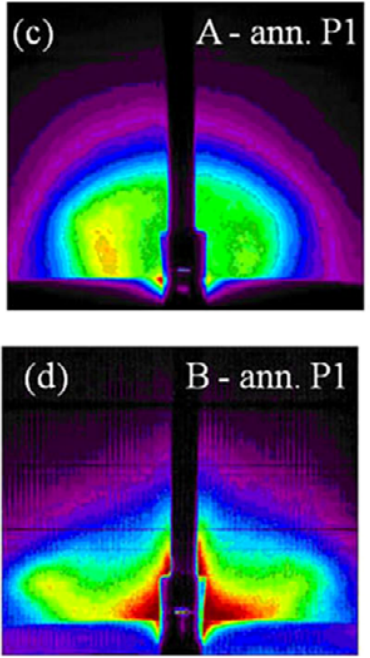
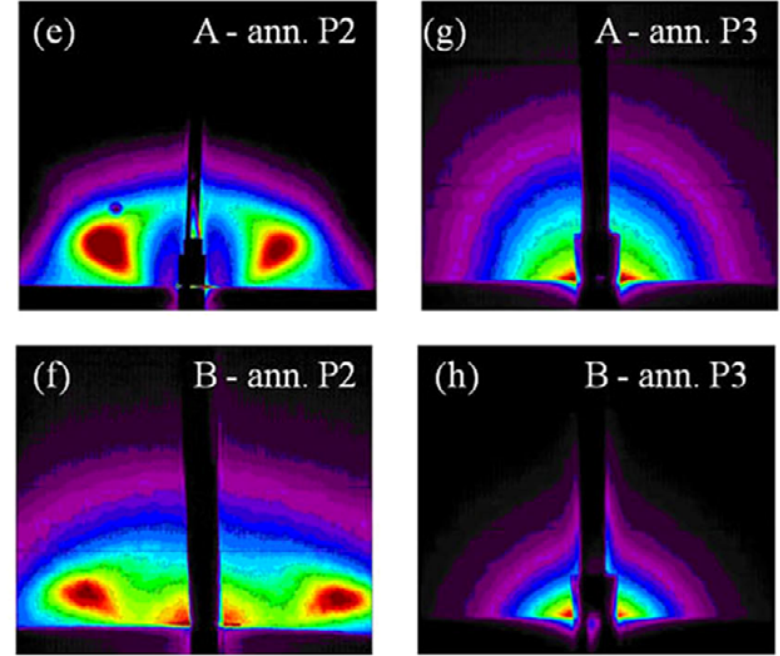

Figure 1. GISAXS maps for as-grown ((a) and (b)) and annealed A and B films ((c)-(h)). The annealing is performed at $800^{\circ} \mathrm{C}$ for $1 \mathrm{~h}$ under different annealing air atmosphere pressures $\left(P_{1}=10^{-5} \mathrm{mbar}, P_{2}=10^{-3} \mathrm{mbar}, P_{3}=P_{\mathrm{atm}}=1000 \mathrm{mbar}\right)$. The axes of the scattering vector $\boldsymbol{Q}\left(Q_{y}\right.$ and $\left.Q_{z}\right)$ are indicated in panel (a).

of the crystalline phases was made using the Joint Committee of Powder Diffraction (JCPDS) database. The spatial distribution study of the $\mathrm{Ge}$ QDs in the $\mathrm{Al}_{2} \mathrm{O}_{3}$ matrix was performed by transmission electron microscopy (TEM) using a Titan 80-300 microscope equipped with a field emission gun working at $300 \mathrm{keV}$, a high angular annular dark field (HAADF) detector for scanning mode (scanning transmission electron microscopy (STEM)) and an image corrector to minimize spherical aberrations. Grazing incidence small angle x-ray scattering (GISAXS) measurements were performed at the synchrotron Elettra Trieste (SAXS beamline) using a photon energy of $8 \mathrm{keV}$ and a two-dimensional (2D) photon detector. The probing $\mathrm{X}$-ray beam lay in the $x z$ plane and the detector was placed perpendicular to the x-ray beam. Details of the measurement geometry are given in [21]. The morphological study of the sample surfaces was performed by atomic force microscopy (AFM) in tapping mode using a commercial Nanoscope III AFM setup in air conditions. The AFM image processing was performed using Nanotec WSxM software [22]. The compositional characterization of the films was performed by the Rutherford backscattering (RBS) technique using a $2.0 \mathrm{MeV} 4 \mathrm{He}^{+}$beam for the identification of the chemical elements present in the films and the variation of their atomic percentage profiles with depth.

\section{Results and discussion}

\subsection{Structural and morphological properties}

Figure 1 presents the GISAXS intensity distributions before and after annealing at different conditions of annealing pressures. The GISAXS maps of the as-grown films (figures 1(a) and (b)) show strong intensity maxima (Bragg spots) indicating the formation of a well ordered array of QDs within the films. The positions and widths of the Bragg spots are related to the parameters of the QD lattice, and give valuable information about the regular arrangement of the QDs and the uniformity of the ordering [21]. The QDs are arranged in a three-dimensional (3D) QD lattice with body centered tetragonal (BCT) structure that can be described by the primitive vectors $a_{1}=(a ; 0 ; 0), a_{2}=(0 ; a ; 0)$ (in the plane parallel to the substrate) and $a_{3}=(a / 2 ; a / 2 ; c)$ embedded in an $\mathrm{Al}_{2} \mathrm{O}_{3}$ matrix. The observed regular ordering is a consequence of the self-assembled growth of the QDs caused by the interplay of diffusion-mediated nucleation and the influence of the growing surface morphology on the positions of the nucleation centers. A detailed study of ordered QD lattice formation during growth is given in our previous work [19], as well as the physical reasons leading to the observed self-assembly. The GISAXS maps were analyzed using the paracrystal model with short range ordering for all basis vectors [21]. The analysis showed that the as-grown films A and B differed by QD sizes and their mutual distances (parameters of the QD lattice), as shown in table 1 together with the data obtained after performing the annealing treatment for different annealing pressures. The GISAXS maps of the annealed films show a very strong dependence of the film structure on the annealing pressure with a similar qualitative behavior for films $\mathrm{A}$ and $\mathrm{B}$. The broadening of the Bragg spots is related to the uniformity of QD ordering, this uniformity being larger as the spots get narrower.

In particular, the films annealed under the lowest air pressure $\left(P_{1}=10^{-5}\right.$ mbar) show distinct Bragg spots (figures 1(c) and (d)), with a similar arrangement to the as-grown films but significantly broader. Thus, we can conclude that the regular ordering still exists in the films annealed at $P_{1}$, but the QDs are shifted from their initial positions leading to a larger disorder degree. Note that, in this case, the intensities of the Bragg spot peaks visible at the right and left sides of the GISAXS maps are slightly different. This difference is due to a small asymmetry in their structure caused by the 

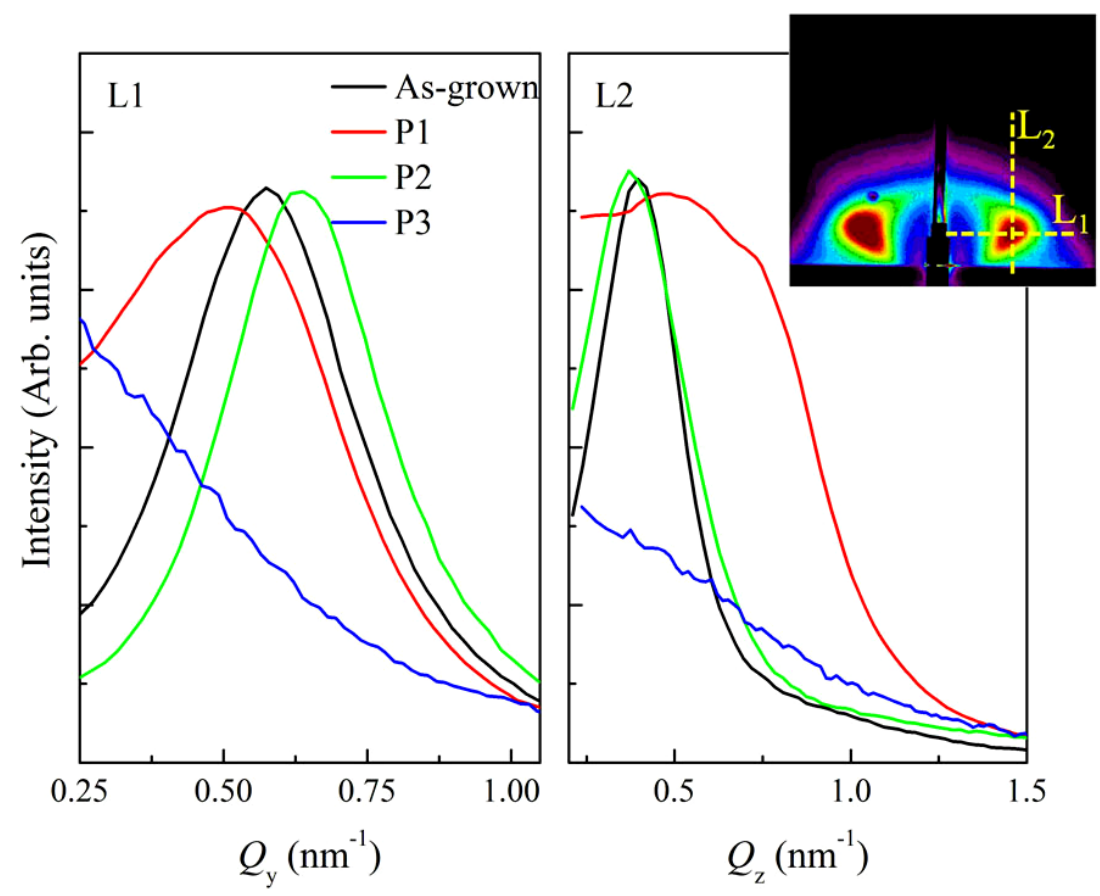

Figure 2. Intensity profiles of the correlation peaks from the GISAXS maps obtained on film A. The profiles are taken from 2D GISAXS maps along the $Q_{y}$ and $Q_{z}$ axes as indicated in the inset of the figure. The annealing pressures are indicated by $P 1\left(P_{1}=10^{-5} \mathrm{mbar}\right), P 2$ $\left(P_{2}=10^{-3}\right.$ mbar $)$ and $P 3\left(P_{3}=P_{\text {atm }}\right)$.

deposition geometry and the use of a fixed substrate stage. It is interesting that regular ordering is entirely preserved for the films annealed at $P_{2}=10^{-3}$ mbar, as shown in figures 1(e) and (f). The Bragg spots for these films are very strong and have practically the same arrangement and widths as the as-grown films. Our previous investigations of film A showed that the Ge was entirely lost for that film, and a void lattice formation with the same arrangement occurred instead [20]. Similar results were obtained for film B.

The GISAXS maps for the films annealed at atmospheric pressure $P_{3}$ (figures 1 (g) and (h)) show a semi-circular feature with no Bragg spots, indicating a complete destruction of the initial regular ordering of QDs, with a random spatial distribution of nearly spherical QDs remaining in the matrix. In this case, the QDs formed in the films $\mathrm{A}$ and $\mathrm{B}$ are much larger with respect to the corresponding as-grown films. Interestingly, the QDs formed after annealing at $P_{3}$ of film B, which had smaller Ge QDs before annealing, are larger than the ones formed in film A (see table 1). The reason may be that the QDs from film B are less stable due to their very small size. Hence, their atoms become free to diffuse at a lower energy and finally they form larger QDs when compared to film A.

Figure 2 shows a comparison of the intensity profiles taken from the GISAXS maps of film A shown in figure 1, through the positions of the Bragg spots. The profiles are taken along the $Q_{y}$ and $Q_{z}$ axes of the reciprocal space, corresponding respectively to the directions parallel and perpendicular to the film's surface. As stated above, the positions of the Bragg spots are related to the arrangement of nano-objects, i.e. the parameters of the QD or void lattices; their widths are related to the ordering quality. From the comparison of the profiles shown in figure 2 it follows that the as-grown film and films annealed at $P_{1}$ and $P_{2}$ have very similar positions of the Bragg peaks. This fact reflects the nearly the same arrangement properties and very similar parameters of the formed nano-object lattices in these films. The peak positions are slightly shifted from each other towards lower or higher $Q_{y}$ and $Q_{z}$ values; however, the differences in the QD lattice parameter values found from the GISAXS analysis are less than $1 \%$. The difference in the peak positions may be a consequence of the non-homogeneity of the as-grown films or the contribution of the nano-object shape and degree of disorder to the GISAXS intensity distribution [21]. The presented observations show that the regular ordering obtained during the growth process exists also after annealing at $P_{1}$ and $P_{2}$ but with a different disorder degree. The film annealed at $P_{3}$ that clearly has no Bragg peaks (i.e. complete destruction of the ordered lattice) is also shown for comparison.

It must be noted that the GISAXS technique is only sensitive to the contrast of the electron density between the matrix and the nano-objects present inside the film. Therefore, it cannot distinguish between Ge QDs and voids due to their significantly different electron density compared with the $\mathrm{Al}_{2} \mathrm{O}_{3}$ matrix. As a consequence, the same GISAXS intensity distribution is observed for both Ge QD and void lattices with a similar spatial arrangement.

The nano-object (Ge QD and void) ordering was confirmed by TEM on as-grown and annealed films (figures 3(a) and (c), respectively). The Fourier transform (FT) analysis of the images shown in the insets clearly demonstrates the regularity in the spatial distribution of the nano-objects and, in agreement with the GISAXS results, the same internal structure for as-grown and annealed films 

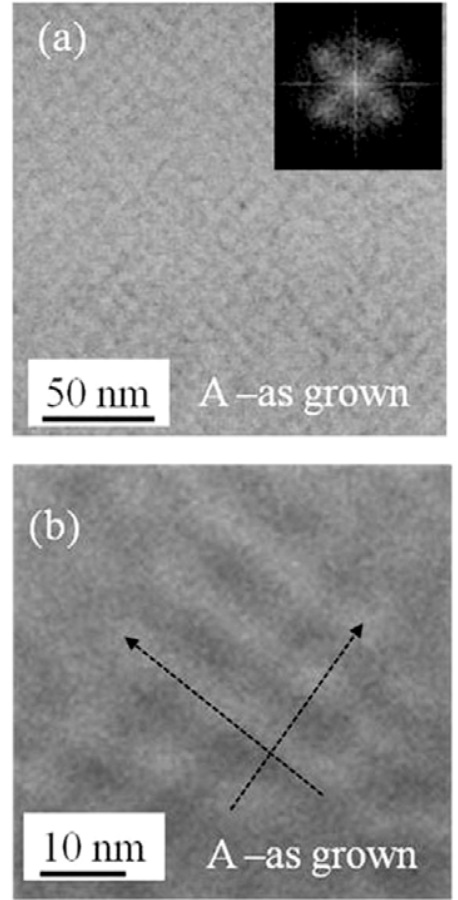
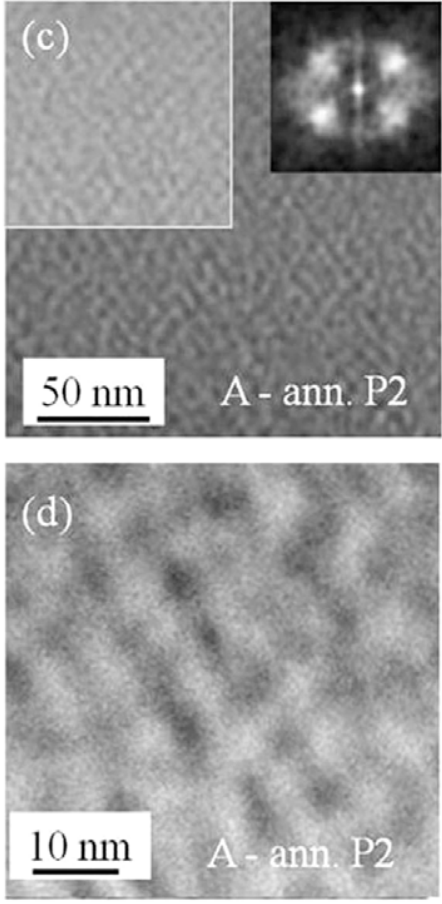
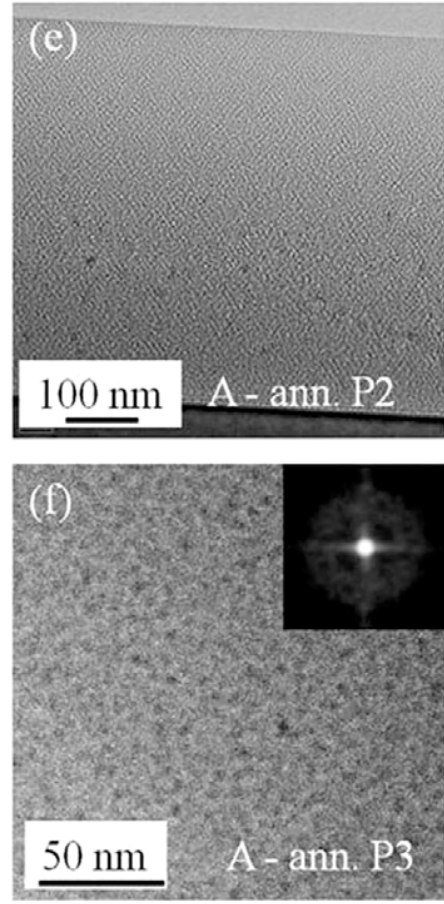

Figure 3. TEM cross-section images of film A. Bright-field TEM (a) and HAADF-STEM (b) images of the as-grown film, where the arrows indicate correlation directions. Bright-field TEM (c) and HAADF-STEM (d) images of the film annealed under $P 2\left(P_{2}=10^{-3} \mathrm{mbar}\right)$. The bright-field TEM image of the whole film cross-section for the annealed film is also reported in (e). The bright-field TEM image of the film annealed under atmospheric pressure $P 3\left(P_{\text {atm }}\right)$ is shown in (f). The insets with black background show the Fourier transforms of the TEM images. The second inset in panel (c) shows the inverted intensity (dark-bright) of the corresponding part of the TEM image.

$\left(P_{2}=10^{-3}\right.$ mbar). By making a comparison between figures 3 (a) and (c), it is possible to observe ordered light and dark circular-like areas, respectively, which indicate ordered nano-objects with different electron densities. In order to distinguish between Ge QDs and voids, HAADF-STEM measurements were performed on these films (figures 3(b) and (d), respectively). Details on the analysis of these measurements together with energy dispersive $x$-ray spectroscopy (EDXS) studies on this kind of sample can be found in our previous work [20]. The bright areas in the STEM image of the as-grown film (figure 3(b)) coincide with the maxima of the Ge concentration (ordered Ge cluster formation), which has a larger scattering cross-section power than $\mathrm{Al}$ and $\mathrm{O}$ atoms. In the case of the annealed film (figure 3(d)), since there is no Ge in the film (as demonstrated below by XRD and RBS measurements), the bright areas coincide with the positions of the $\mathrm{Al}$ and $\mathrm{O}$ concentration maxima, and therefore the dark areas are related to the presence of ordered voids. It is observed that both Ge QDs and voids are nearly spherical in shape but not clearly separated (they touch each other along the directions of their correlation as indicated in figure 3(b)). The RBS analysis of the film annealed at $P_{2}$, presented below in this work, confirms the complete desorption of $\mathrm{Ge}$ from the film, with only $\mathrm{Al}$ and $\mathrm{O}$ atoms remaining. Whether or not the Ge is completely lost from the entire film during annealing, the regular structure of the film is preserved within the whole film thickness, as visible in figure 3(e). The destruction of the regular ordering obtained when the annealing is performed at atmospheric pressure is clearly evidenced in figure 3(f), where no regular pattern is observed in the FT analysis, in agreement with the GISAXS analysis (figures $1(\mathrm{~g})$ and $(\mathrm{h})$ ).

Figure 4 shows the XRD spectra of the films before and after annealing. Typical $\langle 220\rangle$ and $\langle 311\rangle$ Ge diffraction peaks are resolved for the as-grown films. It is well known that the average QD size can be calculated from the full-width-at-half-maximum (FWHM) of the diffraction peaks after performing a Lorentzian curve fitting to the experimental data using the Debye-Scherrer formula [23]. The average Ge QD sizes for the films before and after performing the annealing treatments are shown in table 2. These average size values are slightly smaller than those obtained by GISAXS (see table 1). The differences may arise from the fact that $\mathrm{XRD}$ is sensitive to the size of the crystalline part of the Ge QDs, while GISAXS sees the entire Ge cluster including both the crystalline and the possible amorphous Ge phase. The intensity evolution of the Ge-related diffraction peaks for the annealed samples is also strongly correlated to the annealing pressure. Films $\mathrm{A}$ and $\mathrm{B}$ annealed at $10^{-3}$ mbar show no Ge-related peaks, indicating complete Ge loss from the film, which is in agreement with the formation of a void lattice for these films as measured by GISAXS and HAADF-STEM. The films annealed at $10^{-5}$ mbar show Ge-related peaks with a smaller Ge QD average size with respect to the films annealed at atmospheric pressure that present the largest QDs. This observation is well supported by GISAXS data that showed destruction of the QD lattice and formation of larger Ge clusters. In addition, the intensities of the peaks slightly 


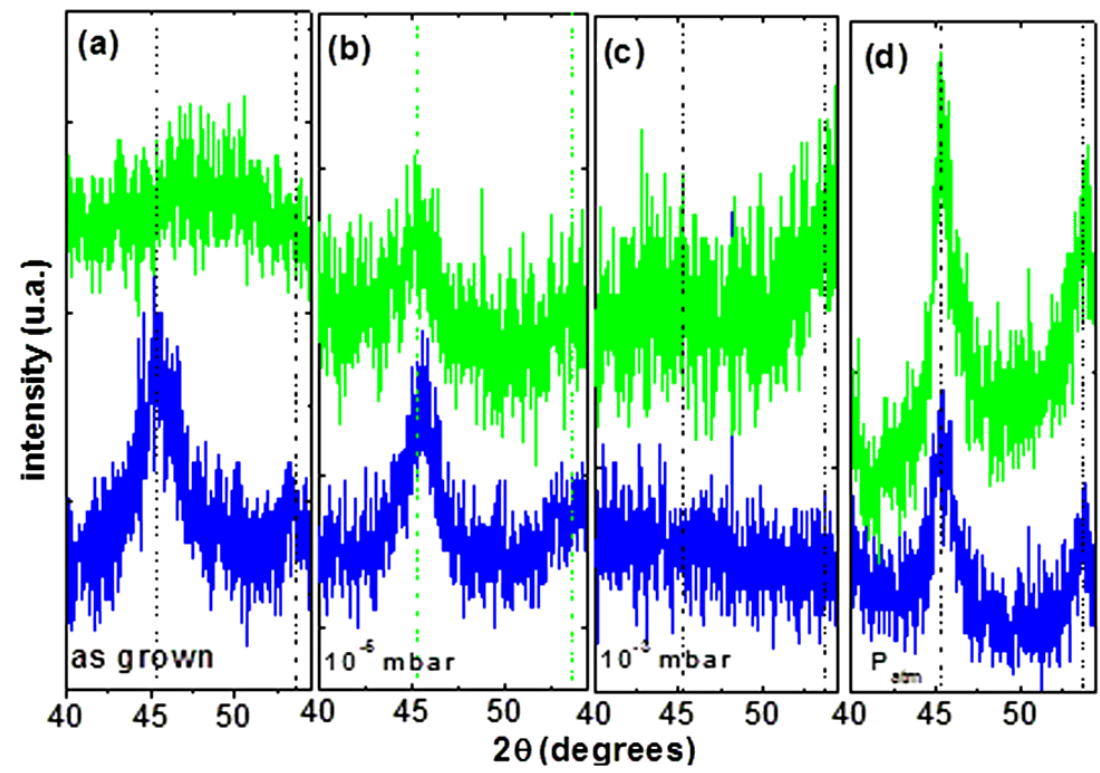

Figure 4. X-ray diffraction spectra for as-grown films (a) and after annealing at $800^{\circ} \mathrm{C}$ for $1 \mathrm{~h}$ at different annealing air pressure values: $10^{-5}$ mbar (b), $10^{-3}$ mbar (c) and atmospheric pressure (d). The vertical dashed lines show the positions of the diffraction peaks corresponding to the $\langle 220\rangle$ and $\langle 311\rangle$ crystallographic planes typical of a Ge cubic crystal structure. The spectra are shifted vertically for a better understanding.

Table 2. Average sizes of Ge QDs for the as-grown and annealed films calculated from the XRD data.

\begin{tabular}{|c|c|c|c|c|}
\hline \multirow[b]{2}{*}{$\begin{array}{l}\text { Sample } \\
\text { name }\end{array}$} & \multirow[b]{2}{*}{$\begin{array}{l}\text { QD size } \\
\text { as-grown (nm) }\end{array}$} & \multicolumn{3}{|c|}{ QD sizes after annealing (nm) } \\
\hline & & $P_{1}=10^{-5} \mathrm{mbar}$ & $P_{2}=10^{-3} \mathrm{mbar}$ & $P_{3}=P_{\mathrm{atm}}$ \\
\hline A & 3.6 & 3.9 & $X$ & 5.1 \\
\hline B & 1.4 & 2.2 & $\mathrm{X}$ & 6.7 \\
\hline
\end{tabular}

increase, suggesting a complete crystallization of the initial Ge clusters. In general, the annealing step helps to enhance not only the crystalline quality but also some diffusion and coalescence of the Ge QDs, leading to a larger crystal size when the annealing is performed at atmospheric pressure.

In figure 5, the surface morphology evolution is shown for different annealing air pressure values as measured by AFM. The surface roughness of the as-grown film (figure 5(a)) presents a root-mean-square (RMS) value of about $0.4 \mathrm{~nm}$. Regions with regularly ordered hill-like structures arranged in a distorted square lattice corresponding to the Ge QD positions may be resolved in the AFM image of the as-grown film measured with high resolution (inset of figure 5(a)). Unfortunately, since the surface roughness of the film increases after performing the annealing treatment, the identification of voids and Ge QD structures on the surface of the annealed films is not straightforward: it is challenging to observe and identify very small features (voids) on surfaces with a relatively high roughness. The strong dependence of the surface roughness on the annealing pressure is depicted in figure 5(e). In particular, a maximum RMS value of about $6 \mathrm{~nm}$ is found when the Ge is entirely lost from the film (annealing pressure of $10^{-3}$ mbar). This result suggests that the surface roughening is related with the loss of the Ge from the film, in agreement with other works [24].

\subsection{Compositional properties}

RBS measurements were performed on film A (qualitatively similar results were found for film B) in order to obtain quantitative information about the atomic species present in the films before and after the annealing treatments with high resolution in depth profiles [25]. In particular, the $\mathrm{Al}_{2} \mathrm{O}_{3}$ stoichiometry and Ge concentration evolution in the film are interesting, since they give relevant information for an understanding of the Ge desorption mechanisms.

Figures 6(a)-(d) show the experimental RBS spectra with the corresponding fitting. The depth concentration profiles of the elements present in the film are presented in figures 6(e)-(h). The analysis of the RBS spectra was performed using the combinatorial optimization simulated annealing (SA) algorithm, which allows one to deconvolute the depth profiles of the different elements [26]. For the as-grown film, the composition profile is homogeneous, with approximately $17 \%$ of Ge atoms. The concentration values in the graph, presented in atomic percentage (\%), are subject to a relative error of $5 \%$. The other two elements ( $\mathrm{O}$ and $\mathrm{Al})$ are also distributed rather uniformly across the films. The concentration ratio of $\mathrm{O}$ and $\mathrm{Al}, c(\mathrm{O}): c(\mathrm{Al})$ in the as-grown films is found to be $c(\mathrm{O}): c(\mathrm{Al})=1.5 \pm 0.1$, very close to the stoichiometry of the ideal $\mathrm{Al}_{2} \mathrm{O}_{3}$ matrix. Therefore, 


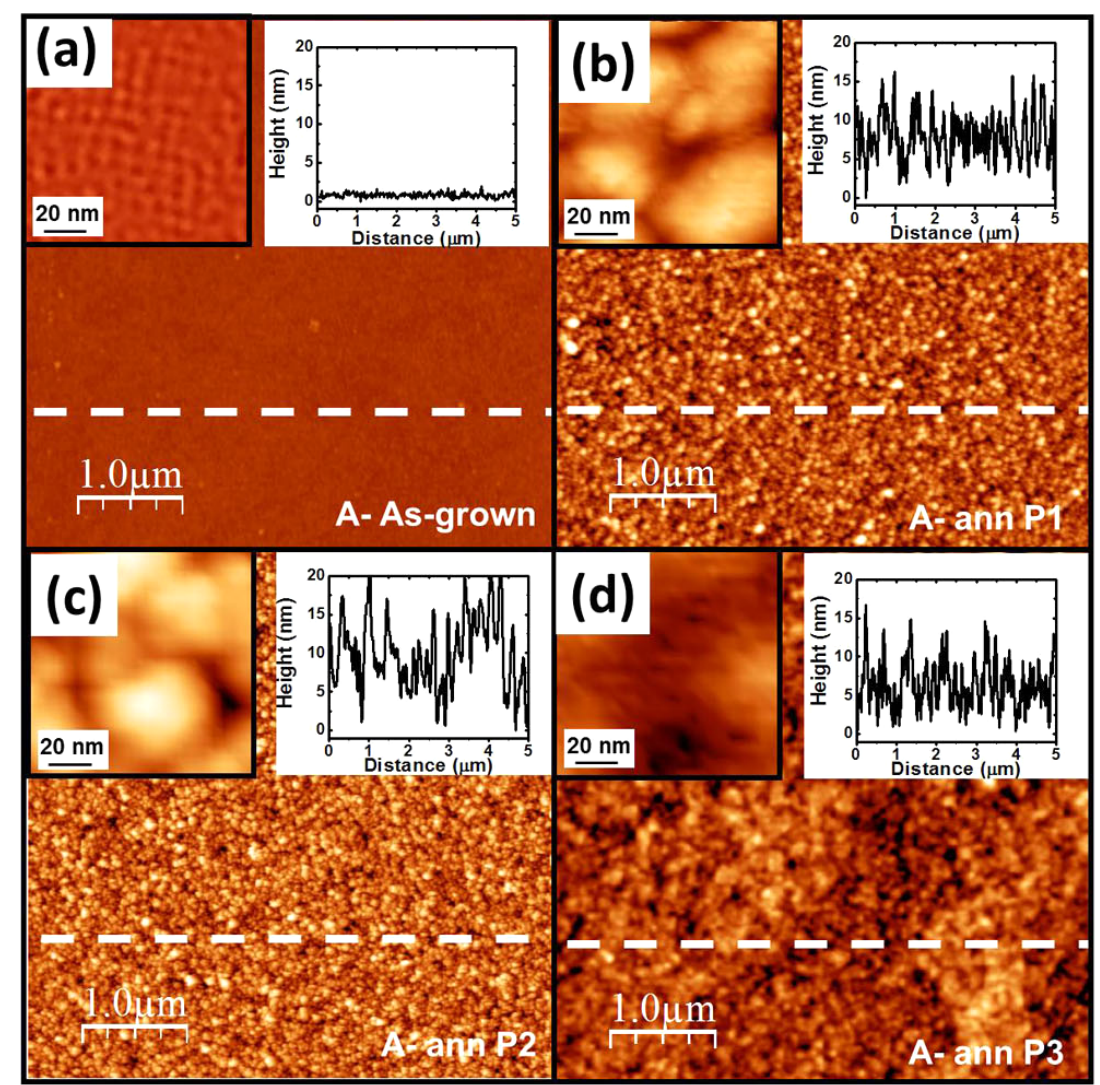

(e)

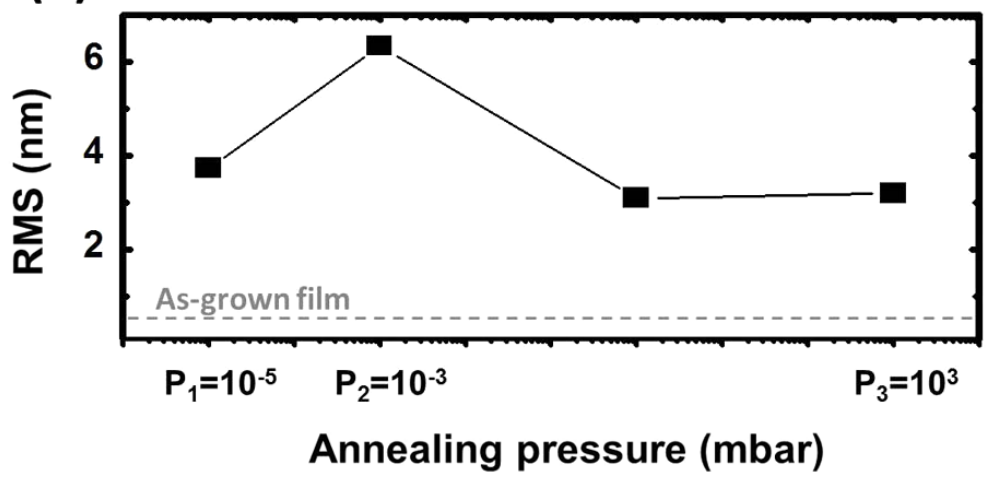

Figure 5. AFM images of the surface roughness of as-grown film $\mathrm{A}$ (a) and after annealing at $800^{\circ} \mathrm{C}$ for $1 \mathrm{~h}$ at different annealing air pressure values: $P 1\left(P_{1}=10^{-5} \mathrm{mbar}\right)(\mathrm{b}) ; P 2\left(P_{2}=10^{-3} \mathrm{mbar}\right)(\mathrm{c}) ; P 3\left(P_{3}=P_{\mathrm{atm}}=1000 \mathrm{mbar}\right)(\mathrm{d})$. The root-mean-square (RMS) values of the surface roughness for the different annealing pressures are reported with a logarithmic scale on the $x$-axis in (e). High resolution AFM images are shown in the insets together with the AFM profiles along the dashed lines. Square-like in-plane ordering of QDs is visible on the surface of the as-grown film (inset to (a)).

practically all $\mathrm{O}$ atoms are bonded to $\mathrm{Al}$ and no significant amount of any other oxygen compound is present in the as-grown film.

The concentration profiles change significantly after the annealing treatments at $P_{2}$ and $P_{3}$ (figures 6(f) and $(\mathrm{g})$ ). In particular, there is some diffusion of $\mathrm{Ge}$ atoms towards the film surface for films annealed at atmospheric pressure, leading to an inhomogeneous Ge concentration profile. In this sample, there is also some excess of oxygen since the $c(\mathrm{O}): c(\mathrm{Al})$ ratio has a value of $2.0 \pm 0.1$, which we attribute to the formation of $\mathrm{Ge}$ oxides from the oxygen coming from the air annealing atmosphere. For the annealing pressure $P_{2}$ (void lattice formation was found in this film), the oxygen excess in the samples decreases and the O:Al ratio value reaches a minimum saturated value of $1.5 \pm 0.1$, whereas the $\mathrm{Ge}$ is completely desorbed from the sample. The $\mathrm{Al}$ and O concentration values are constant through the whole film. Hence, both constant elemental composition and constant structural properties (see figure 3(e)) are found within the entire film. A slightly higher $c(\mathrm{O}): c(\mathrm{Al})$ value $(1.6 \pm 0.1)$ is measured for the sample annealed at $10^{-5}$ mbar, maintaining a similar Ge concentration profile to that of the as-grown film. The dependence of the Ge content on the annealing pressure is explicitly presented in figure 7 . From these results, it is 


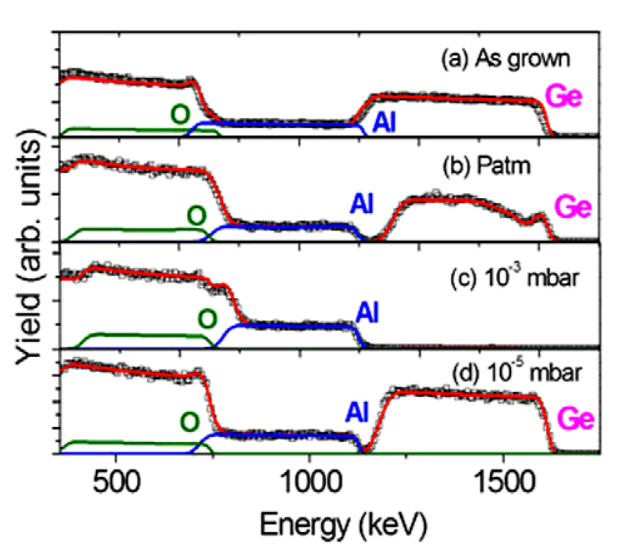

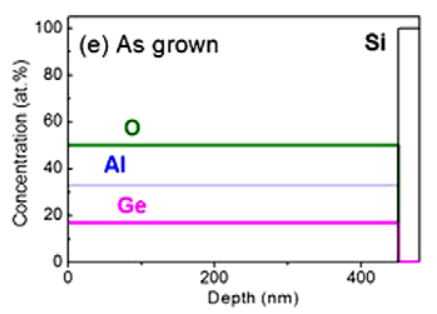

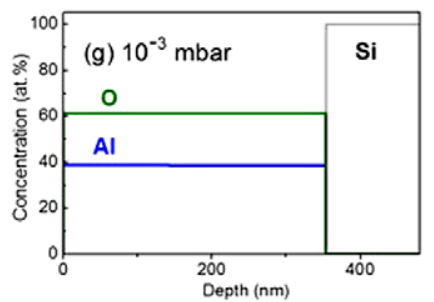

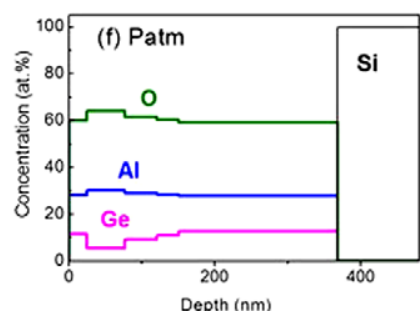

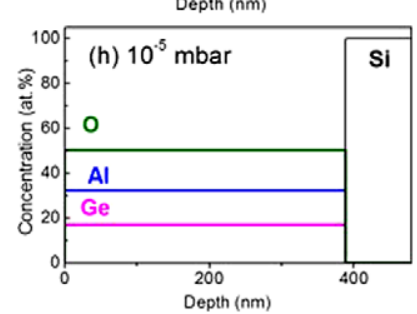

Figure 6. RBS spectra for as-grown film A (a) and after annealing at $800^{\circ} \mathrm{C}$ for $1 \mathrm{~h}$ at different annealing air pressure values (a)-(d). The experimental data are shown with black squares and the fitting to the experimental data with solid red lines. The calculated atomic percentage depth profiles for $\mathrm{O}, \mathrm{Al}$ and $\mathrm{Ge}$ are shown in (e)-(h).

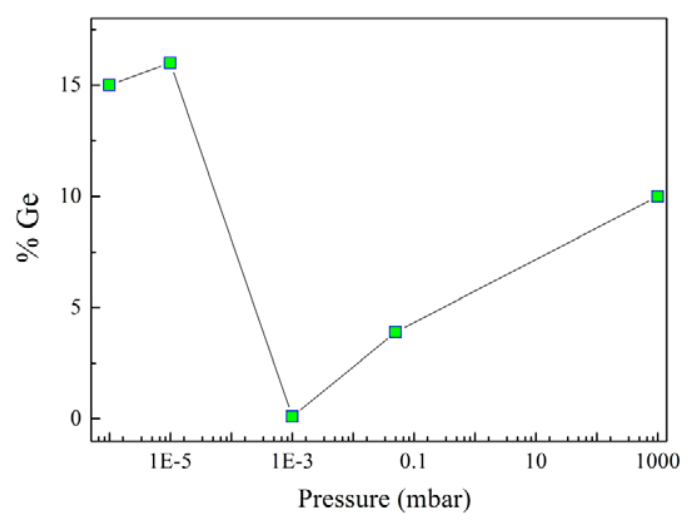

Figure 7. The percentage of Ge atoms measured on film A as a function of the annealing pressure ( $x$-axis logarithmic scale).

clear that there exists some Ge out-diffusion from the film for annealing pressures larger than $1 \times 10^{-5} \mathrm{mbar}$. It can be observed that the Ge concentration in the film decreases as the annealing pressure is increased until a minimum in the Ge concentration in the film is reached (the Ge completely desorbs for a pressure of $1 \times 10^{-3} \mathrm{mbar}$ ). For annealing pressure values larger than $1 \times 10^{-3}$ mbar, the Ge out-diffusion process decreases gradually ( $\mathrm{Ge}$ concentration is increased) as the annealing pressure increases.

The variation of the Ge concentration in the films with the annealing pressure can be explained by considering the oxidation mechanisms of Ge. It has been demonstrated by $\mathrm{X}$-ray photoelectron spectroscopy (XPS) studies that Ge bulk substrates exposed to air oxidize to $\mathrm{GeO}_{2}$ at the surface [27]. On the other hand, the formation of volatile $\mathrm{GeO}$ in oxygen deficient atmospheres through the following reactions has also been reported [28-30]:

$$
\begin{gathered}
\mathrm{Ge}+x \mathrm{H}_{2} \mathrm{O} \rightarrow \mathrm{GeO}_{x}+x \mathrm{H}_{2}(\mathrm{~g}) \uparrow \\
\mathrm{Ge}+\mathrm{GeO}_{2} \rightarrow 2 \mathrm{GeO}(\mathrm{g}) \uparrow \\
2 \mathrm{GeO}+\mathrm{O}_{2}(\mathrm{~g}) \rightarrow 2 \mathrm{GeO}_{2} .
\end{gathered}
$$

In general, Ge oxidation results from the competition between the formation of $\mathrm{GeO}_{2}$ (passive oxidation regime) and $\mathrm{GeO}_{2}$ etching due to $\mathrm{GeO}$ desorption (active oxidation regime), given by reactions (3) and (2), respectively. The evaporation rate of $\mathrm{GeO}$ decreases as the oxygen partial pressure increases due to the formation of stable $\mathrm{GeO}_{2}$ that eventually stops the $\mathrm{GeO}$ evaporation [31]. During the annealing process, crystallization of as-grown Ge clusters together with diffusion of oxygen inside the film to form $\mathrm{GeO}_{2}$ or $\mathrm{GeO}$ takes place. Taking into account our experimental results, it seems clear that there is a competition between the two processes depending on the air pressure used during the annealing process.

In our case, when the annealing process is performed in an oxygen-rich atmosphere (atmospheric pressure), $\mathrm{GeO}_{2}$ compound formation close to the surface of the film is enhanced, stopping thus the desorption of $\mathrm{GeO}$. This is in agreement with the $c(\mathrm{O}): c(\mathrm{Al})$ value of about 2 found in the film where excess oxygen atoms are available for $\mathrm{GeO}_{2}$ compound formation. As the annealing pressure decreases, the $\mathrm{Ge}$ arriving at the surface through diffusion oxidizes to $\mathrm{GeO}_{2}$ at a slower rate, due to a lower oxygen partial pressure, and a higher $\mathrm{GeO}$ desorption rate is expected, which explains the reduction of the Ge amount in the films with decrease of the annealing air pressure. For high vacuum annealing conditions (air pressures $\leq 10^{-5}$ mbar) there are not enough oxidants present in the annealing atmosphere for the formation of a $\mathrm{GeO}_{2}$ layer and the $\mathrm{Ge}$ loss process from the film is inhibited, which explains the relatively high Ge concentration in these films. More questionable is the explanation of the destruction of the QD lattices during annealing at atmospheric pressure. A possible reason for the destruction is the influence of hydrogen from the air atmosphere. It is well known that $\mathrm{H}$ increases the thermal conductivity of the annealing environment, so it is possible that a higher effective temperature is reached for the film annealed at $P_{3}$. The annealing temperature of $800^{\circ} \mathrm{C}$ used in this work is very close to the limiting temperature for 
the destruction of regular ordering when the annealing is performed in vacuum [32]. Thus, a small increase in the temperature could indeed cause the destruction of a regular structure.

Considering the results presented in this paper, special care should be taken when selecting adequate annealing conditions in air of $\mathrm{Ge} / \mathrm{Al}_{2} \mathrm{O}_{3}$ stacks in order to control the $\mathrm{Ge}$ loss rate from the film. It is possible to control the $\mathrm{GeO}$ loss using high vacuum conditions $\left(<10^{-5} \mathrm{mbar}\right)$ or oxygen-rich atmosphere during the annealing treatment $(P \geq 1000$ mbar), in agreement with the findings of Toriumi's group for thin Ge films [33-35].

\section{Conclusion}

In conclusion, we have demonstrated from a structural, morphological and compositional point of view that the vacuum annealing conditions (annealing air pressure) dramatically affect the Ge out-diffusion processes from an amorphous $\mathrm{Al}_{2} \mathrm{O}_{3}$ matrix. Our results show that ordered $\mathrm{Ge}$ QDs/voids in a 3D lattice configuration or disordered $\mathrm{Ge}$ QDs embedded in amorphous $\mathrm{Al}_{2} \mathrm{O}_{3}$ can be obtained as a function of the annealing pressure, independently of the initial QD sizes. We have found that complete Ge QD desorption takes place for a critical annealing air atmosphere pressure of $1 \times 10^{-3}$ mbar. We suggest that this Ge loss is likely to be due to $\mathrm{GeO}$ desorption during the annealing process, where the oxygen partial pressure plays a crucial role in the control of the Ge loss rate.

\section{Acknowledgments}

This study has been partially funded through the projects (i) FEDER funds through the COMPETE program 'Programa Operacional Factores de Competitividade' and by Portuguese funds through the Portuguese foundation for Science and Technology (FCT) in the frame of the project PTDC/FIS/70194/2006 and PEst-C-FIS/UI607/2011-2012; (ii) ELETTRA Synchrotron Radiation Center through the European Community's Seventh Framework Programme (FP7/2007-2013) under grant agreement no 226716. SRCP is grateful for financial support through the FCT grant SFRH/BPD/73548/2010 and JMS is grateful for financial support through the Spanish CSIC JAE-DOC program. MB acknowledges support from the Croatian Ministry of Science, Higher Education and Sport (project number 098-0982886-2859). We thank Dr Anabela Rolo and Engineer José Santos for all the valuable discussions and the sample preparation.

\section{References}

[1] Jabbour G E and Doderer D 2010 Nature Photon. 4 604-5

[2] Konstantatos G and Sargent E H 2010 Nature Nanotechnol. 5 391-400

[3] Stangl J, Holý V and Bauer G 2004 Rev. Mod. Phys. 76725

[4] Chang T C, Yan S T, Liu P T, Chen C W, Lin S H and Sze S M 2004 Electrochem. Solid-State Lett. 7 G17
[5] Konle J, Presting H, Kibbel H, Thinke K and Sauer R 2001 Solid-State Electron. 451921

[6] Lee J J, Wang X G, Bai W P, Lu N and Kwong D-L 2003 IEEE Trans. Electron Devices 502067

[7] Fan J and Chu P K 2010 Small 6 2080-98

[8] Tiwari S, Rana F, Hanafi H, Hartstein A, Crabbé E F and Chan K 1996 Appl. Phys. Lett. 681377

[9] Robertson J 2004 Eur. Phys. J. Appl. Phys. 28265

[10] Deng Z-Y, Fukasawa T, Ando M, Zhang G-J and Ohji T 2001 J. Am. Ceram. Soc. 842638

[11] Gao F, Green M A, Conibeer G, Cho E-C, Huang Y, Wurfl I P and Flynn C 2008 Nanotechnology 19455611

[12] Kita K, Takahashi T, Nomura H, Suzuki S, Nishimura T and Toriumi A 2008 Appl. Surf. Sci. 254 6100-5

[13] Kolobov A, Oyanagi H, Usami N, Toknmitsu S, Hattori T, Yamasaki S, Tanaka K, Ohtake S and Shiraki Y 2002 Appl. Phys. Lett. 80488

[14] Mogaddam N A P, Alagoz A S, Yerci S, Turan R, Foss S and Finstad T G 2008 J. Appl. Phys. 104124309

[15] Rodriguez A, Rodríguez T, Prieto A C, Jiménez J, Kling A, Ballesteros C and Sangrador J 2010 J. Electron. Mater. 391194

[16] Zschintzsch M, Jeutter N M, von Borany J, Krause M and Mücklich A 2010 J. Appl. Phys. 107034306

[17] Kaiser R J, Koffel S, Pichler P, Bauer A J, Amon B, Frey L and Ryssel H 2011 Microelectron. Eng. 88 499-502

[18] Rodriguez A, Rodriguez T, Prieto A C, Jimenez J, Kiling A, Ballesteros C and Sangrador J 2010 J. Electron. Mater. 391194

[19] Buljan M, Pinto S R C, Rolo A G, Martín-Sánchez J, Gomes M J M, Grenzer J, Mücklich A, Bernstorff S and Holý V 2010 Phys. Rev. B 82235407

[20] Pinto S R C, Rolo A G, Gomes M J M, Ivanda M, Bogdanović-Radović I, Grenzer J, Mücklich A, Barber D J, Bernstorff S and Buljan M 2010 Appl. Phys. Lett. 97173113

[21] Buljan M, Radic N, Bernstorff S, Draic G, Bogdanovic-Radovic I and Holy V 2012 Acta Crystallogr. A 68 124-38

[22] Horcas I, Fernandez R, Gomez-Rodriguez J M, Colchero J, Gomez-Herrero J and Baro A M 2007 Rev. Sci. Intrum. 78013705

[23] Langford J I and Wilson A J C 1978 J. Appl. Crystallogr. 11102

[24] Oniki Y, Koumo H, Iwazaki Y and Ueno T 2010 J. Appl. Phys. 107124113

[25] Tesmer J R and Nastasi M 1995 Handbook of Modern Ion Beam Materials Analysis (Pittsburgh, PA: MRS)

[26] Barradas N P, Jeynes C, Homewood K P, Sealy B J and Milosavljevic M 1998 Nucl. Instrum. Methods B 139235

[27] Oh J and Campbell J C 2004 J. Electron. Mater. 33364

[28] Schmidt B, Grambole D and Herrmann F 2002 Nucl. Instrum. Methods Phys. Res. B 191 482-6

[29] Beyer V and Borany J von 2008 Phys. Rev. B 77014107

[30] Markwitz A, Schmidt B, Matz W, Grotzschel R and Mucklich A 1998 Nucl. Instrum. Methods Phys. Res. B 142338

[31] Bernstein R B and Cubicciotti D 1951 J. Am. Chem. Soc. 73 4112-4

[32] Buljan M, Desnica U V, Ivanda M, Radić N, Dubček P, Dražić G, Salamon K, Bernstorff S and Holý V 2009 Nanotechnology 20085612

[33] Wang S, Kita K, Lee C H, Tabata T, Nishimura T, Nagashio K and Toriumi A 2010 J. Appl. Phys. 108054104

[34] Toriumi A, Tabata T, Lee C H, Nishimura T, Kita K and Nagashio K 2009 Microelectron. Eng. 86 1571-6

[35] Wang S K, Kita K, Nishimura T, Nagashio K and Toriumi A 2011 Japan. J. Appl. Phys. 50 04DA01 